\title{
Poisoning of Butanol-Containing Products: Clinical Characteristics and Outcomes
}

\author{
Satariya Trakulsrichai $\mathbb{D}^{1,2}$ \\ Nitchakarn Boonyok ${ }^{1,3}$ \\ Phantakan Tansuwannarat ${ }^{2,4}$ \\ Charuwan Sriapha $\mathbb{D}^{2}$ \\ Winai Wananukul ${ }^{2,5}$ \\ 'Department of Emergency Medicine, \\ Faculty of Medicine Ramathibodi \\ Hospital, Mahidol University, Bangkok, \\ 10400, Thailand; ${ }^{2}$ Ramathibodi Poison \\ Center, Faculty of Medicine Ramathibodi \\ Hospital, Mahidol University, Bangkok, \\ 10400, Thailand; ${ }^{3}$ Emergency Medicine \\ Department, Maharaj Nakhon Si \\ Thammarat Hospital, Nakhon Si \\ Thammarat, 80000, Thailand; \\ ${ }^{4}$ Department of Emergency Medicine, \\ Chakri Naruebodindra Medical Institute, \\ Faculty of Medicine Ramathibodi \\ Hospital, Mahidol University, Bangkok, \\ 10540, Thailand; ${ }^{5}$ Department of \\ Medicine, Faculty of Medicine \\ Ramathibodi Hospital, Mahidol \\ University, Bangkok, 10400, Thailand
}

Correspondence: Satariya Trakulsrichai Department of Emergency Medicine, Faculty of Medicine Ramathibodi Hospital, Mahidol University, Bangkok, I0400, Thailand

Tel +66 2-20I I084

Fax +66 2-20II086

Email satariya.tra@mahidol.ac.th
Background: Butanol (butyl alcohol) is a chemical which occurs naturally in some foods and is used in the manufacture of other chemicals. Current data on butanol poisoning in humans are limited.

Objective: This study describes clinical characteristics and outcomes of patients exposed to products containing butanol.

Methods: We performed a 5-year retrospective cross-sectional study by analyzing data from the Ramathibodi Poison Center Toxic Exposure Surveillance System for 2013-2017.

Results: There were 163 patients included in the study. All products containing butanol reported were agricultural adjuvant products. Most (67.5\%) patients were males and had ingested butanol accidentally (75.5\%). The median age was 42 years. Almost all patients had oral exposure to butanol. At presentation, most of our patients had normal vital signs and were conscious. Clinical presentations mostly included gastrointestinal symptoms $(65 \%)$ and local irritation (28.8\%). Fifty-four patients $(33.1 \%)$ had no obvious clinical effects at presentation. Most patients had normal laboratory tests at presentation, although eight developed systemic effects including high anion gap metabolic acidosis $(n=8)$, acute kidney injury (AKI; $n=5)$, depression of consciousness $(n=5)$, and hypotension $(n=3)$. Of these eight patients, two with intentional ingestion developed altered consciousness, hypotension, AKI, severe metabolic acidosis, and eventually died. One of these died within 1 day after ingestion, while the other died later through complications during admission. Therefore, the mortality rate was $1.23 \%$. Sixty-six patients $(40.5 \%)$ were admitted to hospitals, with a median length of stay of 1 day. Most patients received only supportive treatment and fully recovered.

Conclusion: Agricultural adjuvant products containing butanol or butanol itself caused only mild effects in most patients, but systemic effects occurred in some. The mortality from this poisoning was very low, and both fatalities were from intentional ingestion. Supportive care and proper management of complications should be the main treatment for this form of poisoning.

Keywords: butyl alcohol, agricultural adjuvant products, toxicity, clinical characteristics, outcome

\section{Introduction}

n-butanol (butanol) (also known as 1-butanol or butyl alcohol) is a commodity chemical which is a colorless, flammable liquid with an alcoholic smell. ${ }^{1,2}$ It occurs innately in some foods and is used as an intermediate in the manufacture of other chemicals. ${ }^{1,2}$ Studies in human volunteers and animals have shown that butanol is readily absorbed through the lungs (40\% in humans). It is mainly metabolized via alcohol and aldehyde dehydrogenase in the liver to butyric acid, and is then degraded to shorter acids and ketones, and finally to carbon dioxide. ${ }^{1,2}$ 
In an occupational setting, long-term exposure of workers to high concentrations of butanol vapor was associated with bronchitis, mild anemia, and central nervous system (CNS) effects. ${ }^{1}$ Butanol has been reported to irritate eyes, respiratory tracts, and skin on repeated or prolonged exposures, although the acute oral or parenteral exposure of large doses for therapeutic purposes in humans had no adverse effects. ${ }^{1}$

Current data on oral butanol exposure are limited, and there are few reports of butanol poisoning in humans. ${ }^{3,4}$ One recent case report described a 47-year-old male who had ingested a solvent which was kept in an airport hangar. He had vomited and was found comatose with a Glasgow Coma Scale (GCS) of 3, hypotension and tachycardia. His initial laboratory results showed hypokalemia, acute kidney injury (AKI), metabolic acidosis with elevation of lactate level, and respiratory compromise. Toxicology laboratory tests of his gastric contents and urine sample analyzed by gas chromatography showed the presence of butanol. He was intubated and resuscitated, then finally discharged. $^{3}$

Many cases with a history of exposure to agricultural adjuvant products containing butanol which were used mainly in agricultural area have referred to the Ramathibodi Poison Center (RPC) in Bangkok, Thailand. Therefore, the present study aimed to describe the clinical characteristics and outcomes of cases with products containing butanol exposures in Thailand.

\section{Materials and Methods Study Design}

We performed a retrospective cross-sectional study by analyzing data from the RPC Toxic Exposure Surveillance System for January 2013-December 2017. The primary outcome was the clinical characteristics and outcomes of patients exposed to products containing butanol.

\section{Study Setting and Population}

Our study setting was the RPC, which is a part of a tertiary teaching hospital. Most queries to the RPC are initiated by medical personnel. The RPC received approximately 15,000-20,000 consultations/year. Follow-up telephone calls are performed to collect patient data and the patients' progress, to provide more treatment recommendations, and to ascertain the medical outcomes of cases. All cases are recorded in the RPC Toxic Surveillance System database and are finally reviewed by senior information scientists and clinical toxicologists.

We included all patients who exposed to butanolcontaining products and were referred to the RPC in our study. A diagnosis of poisoning from products containing butanol was based on a history of exposure to butanolcontaining products determined by ingredients listed on the bottles if the container was brought to the hospital or the brand name with detail of ingredients was given by patients. We excluded the patients who co-ingested ethanol, herbs, illicit drugs, pesticides, or other chemicals. The patients with the overdose of pharmaceutical drugs were also excluded.

\section{Study Protocol}

We collected data of all patients including demographics, medical history, clinical data, laboratory test results, management, follow-up details, and the outcomes.

Local effects were accepted as symptoms occurring in areas that were in direct contact with the product; otherwise, they were considered to be systemic effects (which also included hypotension).

Hypotension was defined as a systolic blood pressure (SBP) of $<90 \mathrm{~mm}$ of mercury (mmHg). ${ }^{5}$ Bradycardia and tachycardia were identified as a heart rate of $<60$ and $>100$ beats per minute, respectively. ${ }^{6}$ Fever was determined as a body temperature $>37.7^{\circ} \mathrm{C} .^{7}$ For pediatric patients, the normal vital signs were determined by normal values for each age. ${ }^{8}$

AKI was identified by using Kidney Disease: Improving Global Outcomes clinical practice guidelines (Acute Kidney Injury Network (AKIN) criteria). ${ }^{9}$ We assumed that patients with no underlying disease previously had normal kidney functions previous to the poisoning. Hyponatremia and hypernatremia were determined as serum sodium $<135$ and $>145 \mathrm{mEq} / \mathrm{L}$, respectively. ${ }^{10}$ Hypokalemia and hyperkalemia were determined as serum potassium $<3.5$ and $>5.0 \mathrm{mEq} / \mathrm{L}$, respectively. ${ }^{10}$ Metabolic acidosis was defined as an arterial $\mathrm{pH}<7.40$ together with serum bicarbonate concentration $<24 \mathrm{mEq} / \mathrm{L}$ or when it was noted in the patient's records. ${ }^{11}$ The current range for a normal anion gap is $7 \pm 4 \mathrm{mEq} / \mathrm{L} ;{ }^{11}$ therefore, we defined a high anion gap as $\geq 12 \mathrm{mEq} / \mathrm{L}$.

Excel (Microsoft Corp., Redmond, WA, USA) was used to collect and analyze data in our study. Mean and standard deviation were used for continuous data with a normal distribution. Median with minimum and maximum values was used for non-normally distributed data. 
Categorical data were presented as frequency and percentage.

\section{Results}

One hundred and seventy-four patients were totally identified as having been exposed to products containing butanol. All products containing butanol were agricultural adjuvant products. After patients with co-ingestion had been excluded, a total of 163 poisoned patients were included in our study. Almost all had been exposed by the single oral route $(\mathrm{n}=158)$, although five had been accidentally exposed by other routes including both oral and ocular routes $(\mathrm{n}=1)$, both dermal and ocular routes $(n=2)$, and the dermal route only $(n=2)$. The circumstances of ingestion were accidental exposure for $75.5 \% \quad(n=123)$ and intentional exposure or suicidal attempt for $24.5 \%(n=40)$. All patients in our study informed us of the brand name or details of butanolcontaining products they had been exposed to. All products in our study contained the same ingredients with similar percentages of each ingredient. Butanol was present at $10-20 \%$, and other ingredients included poly (oxy-1,2-ethanediyl), alpha-(4-nonylphenyl)-omegahydroxy-, branched surfactants (CAS number 127087$87-0$ ) at $70-80 \%$, and fatty acids at $1-5 \%$.

Patient general characteristics are summarized in Table 1. The median age of patients was 42 years old, and 39 patients $(23.9 \%)$ were $<15$ years old. Patient clinical presentations are demonstrated in Table 2. Most patients $(65 \%)$ presented with gastrointestinal (GI) symptoms, including nausea or/and vomiting $(\mathrm{n}=99)$, abdominal pain $(n=16)$, and diarrhea $(n=10)$. Neurologic symptoms were described as a depression of consciousness $(n=5)$ and headache $(n=3)$. Respiratory symptoms were dyspnea $(n=4)$ and cough $(n=1)$. Fifty-four patients (33.1\%) had no obvious clinical symptoms or signs at presentation.

Of the four patients exposed to butanol via the dermal route, one developed GI symptoms for only a short period and had no skin irritation before his hospital visit. He also had no GI symptoms after receiving one dose of parenteral antiemetic, so we concluded that he had no systemic effects. The other three patients with skin exposure developed skin irritation and burning lesions. Patients with ocular routes developed eye irritation and/or conjunctivitis after exposure. However, all were eventually discharged after supportive care.
Table I Clinical Characteristics of Patients Exposed to Agricultural Adjuvant Products Containing Butanol

\begin{tabular}{|l|c|}
\hline $\begin{array}{l}\text { Characteristics (Number of Patients with } \\
\text { Data Available) }\end{array}$ & $\mathbf{n}$ (\%) \\
\hline Sex & \\
Male & $110(67.5)$ \\
Female & $53(32.5)$ \\
\hline Age in years, median (min-max) & $42(1-91)$ \\
\hline Region & \\
Northeast & $76(46.6)$ \\
Central & $49(30.1)$ \\
East & $15(9.2)$ \\
North & $9(5.5)$ \\
West & $7(4.3)$ \\
South & $7(4.3)$ \\
\hline Duration from product exposure to hospital & $60(5-1320)$ \\
visit (minutes), median (min-max) (I60 patients) & \\
\hline Amount, median (mL) (min-max) (III patients) & $25(1-300)$ \\
\hline
\end{tabular}

Abbreviations: min, minimum; max, maximum.

Laboratory findings at presentation are shown in Table 3. Most patients had normal results of laboratory tests at presentation.

Of the 163 poisoned patients in our study, eight presented with systemic effects as shown in Table 4. Four of these patients had intentionally ingested, and the others had accidentally ingested butanol. Of the eight patients, one had hyponatremia and another had hypokalemia at presentation. Another patient (Patient 7) had hypotension on arrival at hospital and developed high anion gap metabolic acidosis during hospitalization. Systemic effects included high anion gap metabolic acidosis $(n=8)$, AKI $(\mathrm{n}=5)$, altered consciousness (GCS $<15 ; \mathrm{n}=5$ ), and hypotension $(n=3)$. Five of the eight patients had more than one systemic effect.

Sixty-six patients (40.5\%) were admitted to the hospitals with a median length of stay of 1 day (range, 1 day to $>2$ months). Five patients developed pneumonia as a complication during admission, and three also had systemic effects.

Patients' management was recommended by our RPC; however, the decisions of all treatment were mainly from the treating physicians in each hospital. Most patients were treated with only supportive care and fully recovered. Gastric lavage and activated charcoal administration were given to 34 and 26 patients, respectively. Five patients 
Table 2 Clinical Presentations and Vital Signs of Patients at Presentation

\begin{tabular}{|l|c|}
\hline $\begin{array}{l}\text { Clinical Presentations (Number of Patients } \\
\text { with Data Available) }\end{array}$ & $\mathbf{n}(\%)$ \\
\hline Presenting symptoms* (I63 patients) & \\
$\quad$ No obvious symptoms & $54(33.1)$ \\
Local symptoms & $47(28.8)$ \\
$\quad$-Oral route & $43(26.4)$ \\
-Dermal or ocular routes & $4(2.5)$ \\
Gastrointestinal (GI) symptoms & $106(65.0)$ \\
Neurologic symptoms & $8(4.9)$ \\
Respiratory symptoms & $4(2.5)$ \\
\hline Body temperature $\left({ }^{\circ} \mathrm{C}\right.$, peripheral) (I63 patients) & \\
$<37.8$ & $159(97.5)$ \\
$\geq 37.8$ & $4(2.5)$ \\
\hline Pulse rate (beats/minute) (66 patients) & \\
$60-100$ & $56(84.8)$ \\
$>100$ & $10(15.2)$ \\
\hline Respiratory rate (breaths/minute) (62 patients) & \\
$\leq 20$ & $50(80.6)$ \\
$>20$ & $12(19.4)$ \\
\hline Systolic blood pressure (mmHg) (I63 patients) & \\
$\geq 90$ & $160(98.2)$ \\
$<90$ & $3(1.8)$ \\
\hline Glasgow Coma Scale score (I62 patients) & $4(2.5)$ \\
\hline < 15 & \\
\hline
\end{tabular}

Note: *Some patients presented with > I symptoms.

(3.1\%) were intubated with mechanical ventilator support. Inotropic drugs were given to 4 patients $(2.5 \%)$. Peritoneal dialysis was performed in one $(0.6 \%)$ patient.

Two patients with systemic effects died, so the mortality rate was $1.23 \%$. Both of these patients had intentionally ingested butanol. One was a 70-year-old man (Patient 1 in Table 4) with no previous medical history. He intentionally ingested $45 \mathrm{~mL}$ of an agricultural adjuvant product containing butanol as a suicide attempt about 15 min prior to his hospital visit. After ingestion, he had nausea, vomiting, and diarrhea. At the emergency room (ER), he developed dyspnea and alteration of consciousness. He was intubated and his chest $\mathrm{x}$-ray showed bilateral infiltration. He was diagnosed with ingestion of agricultural adjuvant products containing butanol with aspiration pneumonia and was admitted. Initial laboratory blood test results showed a white blood cell count of $6.8 \times 10^{3} /$ cumm and a calculated anion gap of $16 \mathrm{mmol} / \mathrm{L}$. His arterial blood
Table 3 Laboratory Findings of Patients at Presentation

\begin{tabular}{|l|l|}
\hline $\begin{array}{l}\text { Laboratory Findings (Number of Patients } \\
\text { with Data Available) }\end{array}$ & Levels \\
\hline Serum sodium (mEq/L), mean $\pm \mathrm{SD},(26$ patients) & $139.19 \pm 3.960$ \\
\hline $\begin{array}{l}\text { Serum potassium (mEq/L), mean } \pm \mathrm{SD},(26 \\
\text { patients) }\end{array}$ & $3.88 \pm 0.363$ \\
\hline $\begin{array}{l}\text { Serum chloride (mEq/L), mean } \pm \mathrm{SD},(25 \\
\text { patients) }\end{array}$ & $102.40 \pm 5.008$ \\
\hline $\begin{array}{l}\text { Serum bicarbonate (mEq/L), mean } \pm \mathrm{SD},(26 \\
\text { patients) }\end{array}$ & $22.24 \pm 6.072$ \\
\hline $\begin{array}{l}\text { Anion gap (mEq/L), median (min-max), (24 } \\
\text { patients) }\end{array}$ & $\begin{array}{l}15.00(4.00- \\
33.00)\end{array}$ \\
\hline $\begin{array}{l}\text { Serum creatinine (mg/dL), mean } \pm \mathrm{SD},(23 \\
\text { patients) }\end{array}$ & $1.00 \pm 0.454$ \\
\hline
\end{tabular}

Abbreviations: min, minimum; max, maximum.

gas (ABG) measured $\mathrm{pH}$ 7.0. He developed hypotension with an SBP of $80 \mathrm{mmHg}$ around $17 \mathrm{~h}$ after admission. He was treated intravenously with fluids, norepinephrine, and ceftriaxone ( $2 \mathrm{~g}$ /day). Six days after admission his chest $\mathrm{x}$-ray showed progressive bilateral infiltration. He was still treated with intravenous norepinephrine but his antibiotic treatment changed to intravenous piperacillin-tazobactam, then finally meropenem. Ten days after admission, he was stuporous (GCS: E1VtM5), and intravenous vancomycin was given. He became comatose and developed bradycardia and hypotension, and eventually died on the fifteenth day of hospitalization.

The second patient who died was a 76-year-old man (Patient 8 in Table 4) who intentionally ingested about $20 \mathrm{~mL}$ of butanol around $2 \mathrm{~h}$ before his hospital visit. After ingestion, he developed nausea and vomiting. At the ER, gastric lavage was performed, and he received activated charcoal. He then became drowsy with a GCS of 9 (E2V2M5) and hypotension. He was treated with volume replacement therapy, intubated, and referred to a tertiary hospital. Initial laboratory blood test results showed a white blood cell count of $11.18 \times 10^{3} /$ cumm and a calculated anion gap of $25.2 \mathrm{mmol} / \mathrm{L}$. His initial ABG measured $\mathrm{pH}$ 6.97, partial pressure of oxygen was $218 \mathrm{mmHg}$, partial pressure of carbon dioxide was 24 $\mathrm{mmHg}$, and oxygen saturation was $99 \%$. After admission, he still had severe metabolic acidosis (ABG pH 6.94, serum bicarbonate $7.6 \mathrm{mmol} / \mathrm{L}$ with a calculated anion gap of $21.2 \mathrm{mmol} / \mathrm{L}$ from serum electrolytes), and 


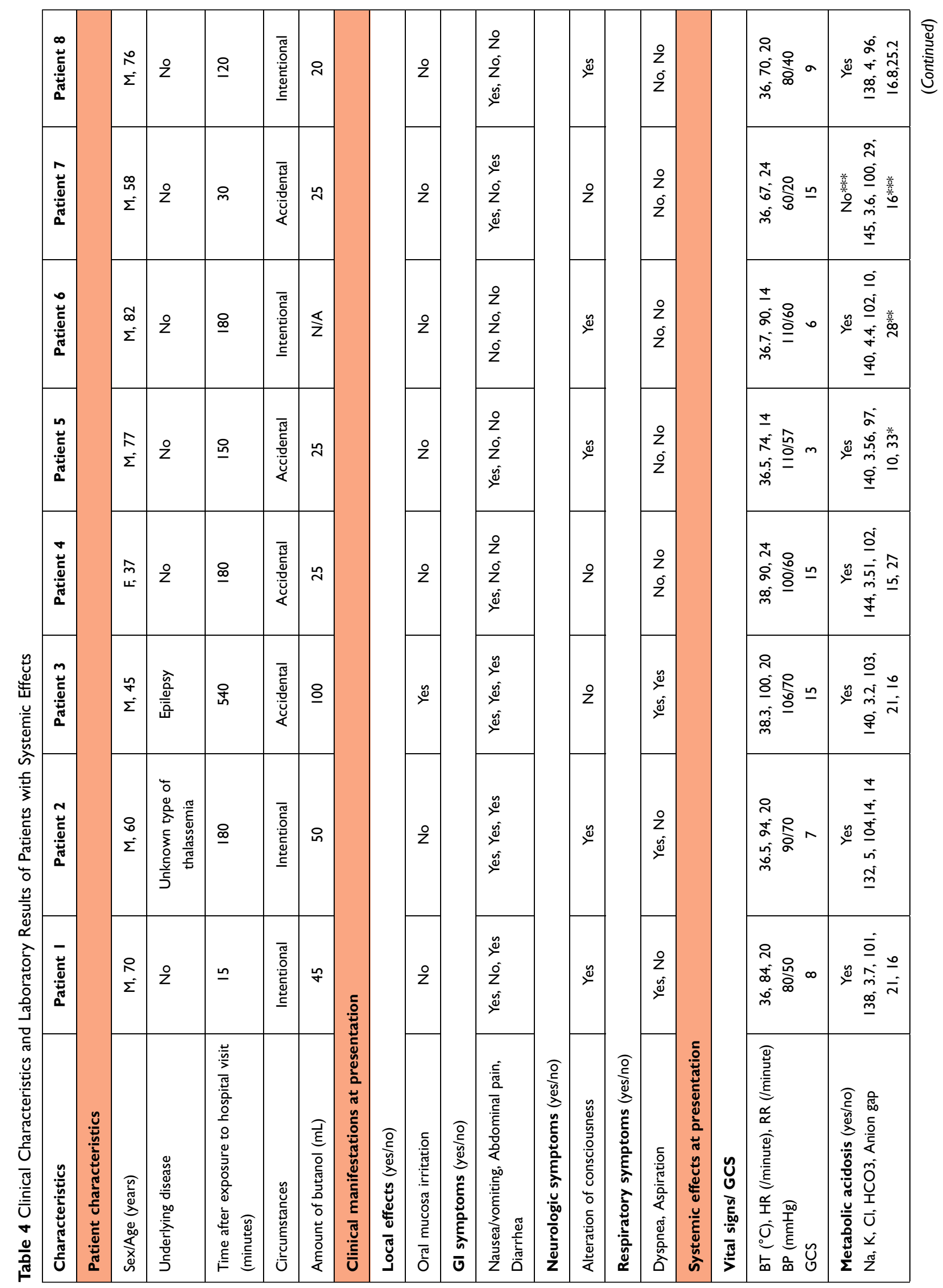




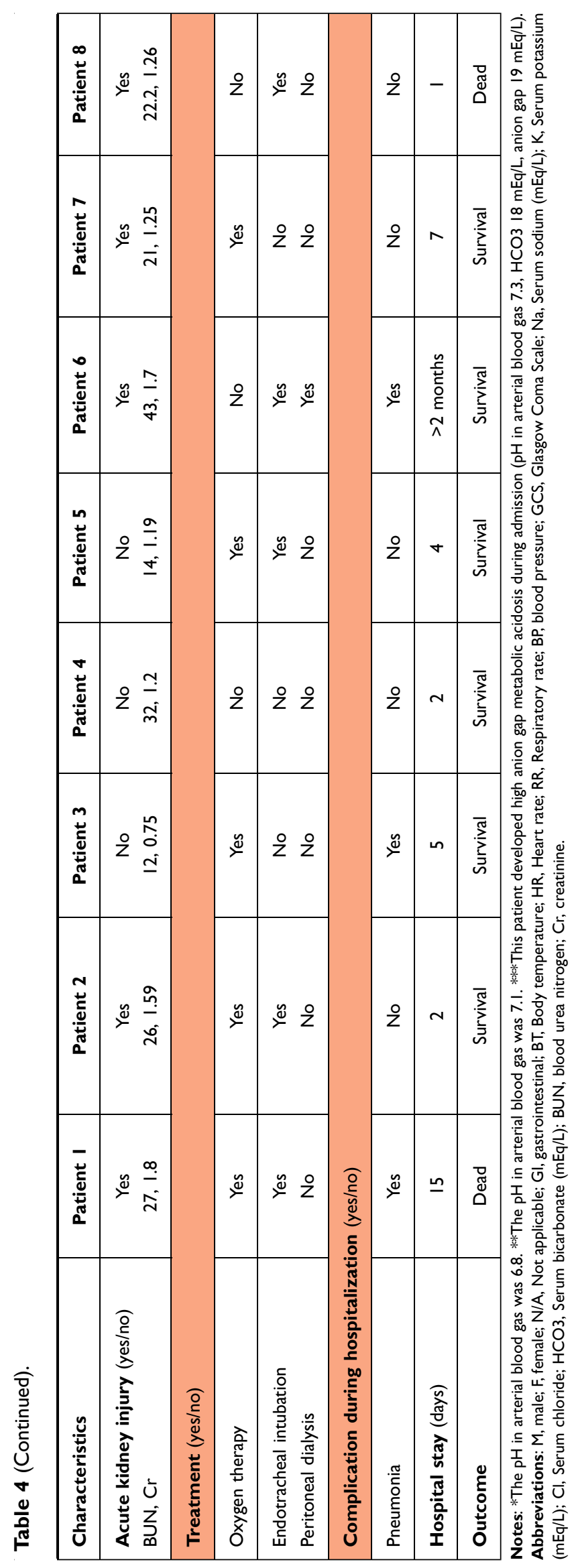

developed hypotension. He was treated with intravenous fluids, norepinephrine, and dopamine, but his symptoms worsened, he went into cardiac arrest, and eventually died within 1 day after ingestion. He was diagnosed with intoxication by ingestion of an agricultural adjuvant product containing butanol.

\section{Discussion}

The agricultural adjuvant products containing butanol in our study were formulated with the main compounds as surfactants and other co-formulants as fatty acids. Therefore, the observed local effects such as GI symptoms and systemic effects could be attributed, in part, to these coformulants. However, although hepatotoxic adverse effects or toxicities have been noted for poly(oxy-1,2-ethanediyl), alpha-(4-nonylphenyl)-omega-hydroxy-, branched, ${ }^{12}$ hepatotoxicity was not observed in our patients. Fatty acids were present as co-formulants at very low levels $(1-5 \%)$, so it is possible that these two ingredients did not contribute to the observed clinical effects in our patients. However, further observation of the toxicity of these compounds is required to ascertain their clinical effects in humans.

The most common presentations were GI symptoms such as nausea and/or vomiting. The observed systemic effects included high anion gap metabolic acidosis, AKI, depression of consciousness, and hypotension, which were consistent with the findings of a previous case who vomited and developed these systemic effects after butanol ingestion. ${ }^{3}$ Taken together, the presenting clinical effects and main systemic effects reported in our study were consistent with the effects reported from butanol poisoning. ${ }^{3}$ Therefore, we postulate that the effects observed in our patients mainly derived from butanol toxicity. Butanol poisoning by oral exposure in humans is rarely reported in the literature, and to our knowledge only a few cases have been documented. ${ }^{3,4}$ Therefore, this study is one of the largest studies on poisoning by products containing butanol reported to date.

Our patients were consulted and referred from every region of Thailand. Most were from the northeast which is largely agricultural and where agricultural products are widely used. Approximately two-thirds of our patients were male, and the median age was 42 years. Most patients were exposed to butanol accidentally. We postulate that the containers of these products were easily accessible or that the products were kept in other containers, making them readily available for accidental ingestion. Additionally, poor labeling or a lack of labeling on 
the agricultural adjuvant product bottles could have prevented a clear understanding of their contents. Therefore, health education to make individuals aware of the toxicity of agricultural products would play an important role in preventing this poisoning.

Most patients had no symptoms or only mild symptoms and signs, and most had normal vital signs and laboratory findings at presentation and during hospitalization. Therefore, this poisoning, especially from oral exposure, appears to cause only minor effects. For the systemic effects observed, metabolic acidosis might be partly explained by the accumulation of butanol metabolites in the formation of butyric acid. ${ }^{1,2}$ A study in rats demonstrated that systolic and diastolic blood pressures transitorily decreased following butanol exposure, ${ }^{13}$ so hypotension would be an expected cardiovascular effect in patients with severe butanol poisoning. Oral or inhalation exposure to butanol resulted in neurotoxic effects in animal models, which might be explained by involvement of the N-methyl-D-aspartate (NMDA) glutamate receptor and the gamma-aminobutyric acid (GABA) receptor. ${ }^{1,2}$ This could account for the observed CNS depression in our patients. AKI identified in our patients could be partially explained by fluid loss from GI symptoms. The resulting AKI by butanol poisoning needs further study to elucidate the pathophysiology of this toxicity. Therefore, in addition to the observation and monitoring of vital signs, consciousness, and clinical effects, we suggest that serum electrolyte and kidney function should be investigated in patients exposed to butanol.

Two patients died in our study. Both had deliberately ingested butanol and developed alteration of consciousness, hypotension, AKI, and severe metabolic acidosis. One died within 1 day after ingestion, which was consistent with a previous case showing rapid and severe clinical effects after butanol exposure, ${ }^{3}$ while the other died later through complications. We recommend that patients who intentionally ingest butanol, or who show abnormal consciousness or hypotension should be closely observed, monitored, and treated, especially in the first $24 \mathrm{~h}$ after ingestion. Complications during admission should also be aggressively and properly managed.

In the general management of this poisoning, supportive care and the correct management of complications should be the main treatment for all patients. However, we suggest that intensive monitoring and aggressive treatment including the management of complications during hospitalization should also be implemented for patients with systemic effects. Hemodialysis and the intravenous administration of ethanol as treatments for this poisoning have not been extensively studied so their benefits are unclear; therefore, further studies, particularly in severely affected patients, are warranted.

Our study had some limitations listed as follows. First, reporting cases who exposed to agricultural adjuvant products containing butanol to the RPC is not compulsory. Therefore, not all cases with product exposures are referred to our institution, particularly mild cases. So, it is possible that the mortality rate and the rate of severe poisoning might be different from the rates reported in our study. Second, the retrospective nature of the study may have resulted in missing or incomplete or unclear data. Third, the diagnosis of poisoning was mainly based on a history of product exposure, and butanol or its metabolites could not be analyzed in our patients' blood or urine to confirm the diagnosis. Finally, some laboratory blood tests such as arterial blood gas analysis were not available and could not be analyzed due to the limited resources of laboratories in some hospitals in Thailand.

\section{Conclusions}

Agricultural adjuvant products containing butanol or butanol itself were found to mainly cause only mild effects in most patients in this study, but systemic effects occurred in some. The mortality from this poisoning was very low and both fatalities occurred from intentional ingestion. Supportive care and proper management of complications are the main treatments for butanol poisoning in all patients.

\section{Ethical Approval}

This study was approved by the Institutional Ethics Committee Board of Ramathibodi Hospital Faculty of Medicine, Mahidol University (approval number COA. MURA2018/221). Because this study was a retrospective cross-sectional study which used a pre-existing confidential database from the RPC, patient consent was not needed by our hospital's ethics committee board. This study was conducted in accordance with the Declaration of Helsinki.

\section{Disclosure}

The authors report no conflicts of interest for this work. 


\section{References}

1. European Centre for Ecotoxicology and Toxicology of Chemicals. n-Butanol (CAS No. 71-36-3). Brussels: ECETOC; 2003. (JACC No. 41). Available from: https://www.ecetoc.org/wp-content/uploads/ 2014/08/JACC-041.pdf. Accessed November 16, 2020.

2. Segal D, Bale AS, Phillips LJ, et al. Issues in assessing the health risks of n-butanol. $J$ Appl Toxicol. 2020;40:72-86. doi:10.1002/jat.3820

3. Bunc M, Pezdir T, Mozina H, Mozina M, Brvar M. Butanol ingestion in an airport hangar. Hum Exp Toxicol. 2006;25(4):195-197. doi:10.1191/0960327106ht607oa

4. Levkov IA, Vullo II. [A case of butyl alcohol poisoning]. Gig Tr Prof Zabol. 1965;9:56-57. Russian.

5. Nicks BA, Gaillard JP. Approach to nontraumatic shock. In: Tintinalli JE, Ma O, Yealy DM, editors. Tintinalli's Emergency Medicine: A Comprehensive Study Guide. 9th ed. New York, NY: McGraw-Hill Education; 2020:57-63.

6. Michaud GF, Stevenson WG. Physiologic and nonphysiologic sinus tachycardia. In: Jameson J, Fauci AS, Kasper DL, editors. Harrison's Principles of Internal Medicine. 20th ed. New York, NY: McGraw-Hill Education; 2018:4316-4318.

7. Ogoina D. Fever, fever patterns and diseases called 'fever'-a review. $J$ Infect Public Health. 2011;4:108-124. doi:10.1016/j. jiph.2011.05.002
8. Nelson LS, Howland MA, Lewin NA, et al. Initial evaluation of the patient: vital signs and toxic syndromes. In: Nelson LS, Howland MA, Lewin NA, et al. editors. Goldfrank's Toxicologic Emergencies. 11th ed. New York: McGraw Hill Education; 2019:28-31.

9. Kellum JA, Lameire N, Aspelin P, et al. Kidney disease: improving global outcomes (KDIGO) acute kidney injury work group. KDIGO clinical practice guideline for acute kidney injury. Kidney Int Suppl. 2012;2:1-138.

10. Mount DB. Fluid and electrolyte disturbances. In: Jameson J, Fauci AS, Kasper DL, editors. Harrison's Principles of Internal Medicine. 20th ed. New York, NY: McGraw-Hill Education; 2018:789-817.

11. Charney AN, Hoffman RS. Fluid, electrolyte, and acid-base principles. In: Nelson LS, Howland MA, Lewin NA, editors. Goldfrank's Toxicologic Emergencies, 11th ed. New York: McGraw Hill Education; 2019:189-202.

12. Haz-Map. Poly(oxy-1,2-ethanediyl), alpha-(4-nonylphenyl)-omegahydroxy-, branched. Available from: https://haz-map.com/Agents/ 16680? referer=BrowseByCategory\&return_url=\%2FAgents $\% 2 \mathrm{~F}$ (BrowseByCategory)\%2F10\%3Fsub_cat_id\%3DEmulsifiers\% 252FSurfactants. Accessed December 3, 2020.

13. Geppert E, Sturz J, Haase W, Isselhard W. [The effect of n-butanol on the metabolic status of some organs of rats and the circulation of rabbits]. Arzneimittelforschung. 1976;26:1333-1337. German.
International Journal of General Medicine

\section{Publish your work in this journal}

The International Journal of General Medicine is an international, peer-reviewed open-access journal that focuses on general and internal medicine, pathogenesis, epidemiology, diagnosis, monitoring and treatment protocols. The journal is characterized by the rapid reporting of reviews, original research and clinical studies

\section{Dovepress}

across all disease areas. The manuscript management system is completely online and includes a very quick and fair peer-review system, which is all easy to use. Visit http://www.dovepress.com/ testimonials.php to read real quotes from published authors. 